TAMKANG JOURNAL OF MATHEMATICS

Volume 33, Number 3, Autumn 2002

\title{
AUTOMORPHISM GROUPS OF COMPLEX ANALYTIC GROUPS
}

\author{
P. B. CHEN AND T. S. WU
}

\begin{abstract}
Let $G$ be a faithfully representable complex analytic group and Aut $G$ the group of all complex analytic automorphisms of $G$. We prove that the identity component of Aut $G$ can be identified as an algebraic group.
\end{abstract}

\section{Introduction}

If $H$ is a connected complex affine algebraic group, in [4], G. Hochschild and G. D. Mostow prove that the connected component of the neutral element in the group of all rational automorphisms of $H$ is algebraic. It is our main purpose here to seek a reasonable analogous result in the category of complex analytic groups. More precisely, if $G$ is a faithfully representable complex analytic group, we show that the connected component $(\text { Aut } G)_{\circ}$ of the neutral element in the group of all complex analytic automorphisms of $G$ can be identified as an algebraic group. Furthermore, as an application, we show that the fixer of an element of $G$ in $(A u t G)$ 。 can also be identified as an algebraic group.

Notations. Let $G$ be a group. If $f$ is a function on $G$ and $H$ is a subset of $G$, we denote by $f \mid H$ the restriction of $f$ to $H$. If, in addition, $G$ is a Lie group and $K$ is a subgroup of $G$, we denote by $L(G)$ the Lie algebra of $G$ and by $K_{\circ}$ the connected component of $K$ that contains the identity element. We denote by $\mathbf{C}^{*}$ the multiplicative group of nonzero complex numbers.

\section{Preliminaries.}

Let $G$ be a faithfully representable complex analytic group. If $f$ is a function on $G$ and $x \in G$, we define the left (resp. right) translate $x \cdot f$ (resp. $f \cdot x)$ of $f$ by $x$ to be $(x \cdot f)(y)=f(y x)$ (resp. $(f \cdot x)(y)=f(x y))$. We denote by $R(G)$ the Hopf algebra of all complex analytic functions $f$ of $G$ into $\mathbf{C}$ for which the complex vector space spanned by all left translates of $f$ is finite-dimensional. Let $S \subset R(G)$. We say that $S$ is left (resp. right) stable if $S$ is stable under all left (resp. right) translations. We say that $S$ is bistable if $S$ is both left stable and right stable. An element $f$ of $R(G)$ is

Received June 14, 2001

2000 Mathematics Subject Classification. Primary 22D45, 22E55.

Key words and phrases. Faithfully representable complex analytic groups, automorphism groups, algebraic groups, representative function. 
called semisimple if the representation of $G$ by left translations on the finite-dimensional space spanned by all left translates of $f$ is semisimple. If $B$ is a subset of $R(G)$, we use $B_{s}$ to denote the collection of all semisimple functions in $B$. Let $\operatorname{Hom}(G, \mathbf{C})$ be the collection of all complex analytic homomorphisms of $G$ into the additive group $\mathbf{C}$. Let $Q=\{\exp \circ f: f \in \operatorname{Hom}(G, \mathbf{C})\}$, where exp:C $\rightarrow \mathbf{C}^{*}$ is the exponential map. A subalgebra $B$ of $R(G)$ is called a basic subalgebra if elements of $Q$ is free over $B$ and $R(G)=B[Q]$. A normal basic subalgebra $B$ of $R(G)$ is a left stable basic subalgebra such that $B_{s}$ is bistable. A subalgebra of $R(G)$ is called fully stable if it is bistable and is stable under the antipode $\eta: R(G) \rightarrow R(G)$ sending every element $f$ of $R(G)$ onto the function defined by $\eta(f)(x)=f\left(x^{-1}\right)$. A subalgebra of $R(G)$ is called a regular subalgebra if it is fully stable and finitely generated as a $\mathbf{C}$-algebra, and contains a normal basic subalgebra of $R(G)$.

Now, let $K$ be a nucleus of $G$. Then there exists a normal basic subalgebra $B$ of $R(G)$ such that $K$ is the kernel of the representation of $G$ by left translations on $B_{S}$ ([2, Theorem 1.1]), and there is a unique regular subalgebra $R(G, K)$ of $R(G)$ such that, if $B$ is any normal baic subalgebra of $R(G)$ associated with $K$ as above, then $R(G, K)$ coincides with the smallest fully stable subalgebra of $R(G)$ that contains $B$. We call $R(G, K)$ the nuclear subalgebra of $R(G)$ that is associated with $K$ ([3, Section 5]). Put $S=R(G, K)$. Let $A_{S}$ be the group of all proper automorphisms of $S$ (that is, algebra automorphisms of $S$ that commute with right translations) and $G_{S}$ the group of all left translations on $S$. Since every normal basic subalgebra separates points of $G$, we may identify $G$ with $G_{S}$ via the map sending every element $x$ of $G$ onto the restriction of the left translation by $x$ to $S$. By [3, Theorem 3.1], $A_{S}$ is an algebraic group as well as a complex analytic group, $G$ is closed and algebraically dense in $A_{S}$, and $A_{S}$ and $G$ share the same commutative subgroup. What follows are the statements of two results from [1] that will be used later.

Lemma 1.1.([1, Lemma 1.1(1)]) Let $G$ be a faithfully representable complex analytic group. Let $K$ be a nucleus of $G$ and $S$ the associated nuclear subalgebra of $R(G)$. If $U$ is the unipotent radical of $A_{S}$ and $A$ is the maximal reductive complex Lie subgroup of the center of $A_{S}$, then $U \cdot A$ is the maximal nilpotent normal subgroup of $A_{S}$.

Theorem 1.2.([1, Theorem 2.5]) Let $G$ be a faithfully representable complex analytic group and $A$ the maximal reductive complex Lie subgroup of the center of $G$. Then there is a nucleus $K$ of $G$ such that $\theta(K) \subseteq K A_{\circ}$ for all $\theta \in$ Aut $G$.

\section{Automorphism Groups.}

From now on, all notations will be fixed. Let $G$ be a faithfully representable complex analytic group and Aut $G$ the gruop of all complex analytic automorphisms of $G$. Identifying with a subgroup of $G L(L(G))$, Aut $G$ has a natural structure of a complex Lie group.

Let $K$ be a fixed nucleus of $G$. Let $N$ be the representation radical of $G$ (that is, the intersection of the kernels of all semisimple representations of $G$ ) and $A$ the maximal 
reductive complex Lie subgroup of $Z(G)$. Let $\operatorname{Aut}(G, K)$ be the closed complex Lie subgroup of Aut $G$ that consists of all those $\theta \in A u t G$ for which $\theta(K) \subset K A_{\circ}$. Let $W(G, K)$ be the closed comple Lie subgroup of Aut $G$ that consists of all those elements in Aut $G$ that leave $K$ invariant, and let $\operatorname{Hom}_{N}\left(K, A_{\circ}\right)$ be the group of all complex analytic homomorphisms of $K$ into $A_{\circ}$ that are trivial on $N$.

Let $\tau \in \operatorname{Hom}_{N}\left(K, A_{\circ}\right)$. Fix a maximal reductive complex Lie subgroup $H$ of $G$. Define $\tau^{+}: G \rightarrow G$ by $\tau^{+}(k h)=k h \tau(k)$ for all $k \in K$ and $h \in H$. From the facts that $\tau$ is trivial on $N$ and $[G, K] \subset N$, one checks readily that $\tau^{+} \in \operatorname{Aut}(G, K)$. Because any two maximal reductive complex Lie subgroups of $G$ are conjugate by elements of $N$, all maximal reductive complex Lie subgroups of $G$ are contained in $H N$. Since $\tau^{+}$is trivial on $H N$, the definition of $\tau^{+}$above is independent of the choice of $H$.

Let $V(G, K)=\left\{\tau^{+} \in \operatorname{Aut}(G, K): \tau \in \operatorname{Hom}_{N}\left(K, A_{\circ}\right)\right\}$. If $\gamma, \sigma \in \operatorname{Hom}_{N}\left(K, A_{\circ}\right)$ and $\rho(k)=\gamma(k)^{-1}$ for all $k \in K$, one checks directly that $\gamma^{+} \circ \sigma^{+}=(\gamma \sigma)^{+}, \rho \in \operatorname{Hom}_{N}\left(K, A_{\circ}\right)$ and $\rho^{+}=\left(\gamma^{+}\right)^{-1}$. It follows that $V(G, K)$ is a closed complex Lie subgroup of $\operatorname{Aut}(G, K)$. Now, we are ready to describe a semidirect product decomposition of $\operatorname{Aut}(G, K)$.

Theorem 2.1. Let $G$ be a faithfully representable complex analytic group and $K$ a nucleus of $G$. Then Aut $(G, K)$ is a semidirect product $V(G, K) \cdot W(G, K)$ with $V(G, K)$ being normal in $\operatorname{Aut}(G, K)$.

Proof. Let $\alpha \in \operatorname{Aut}(G, K)$. By definition, $\alpha$ determines uniquely two maps $\beta$ : $K \rightarrow K$ and $\tau: K \rightarrow A_{\circ}$ such that $\alpha(k)=\beta(k) \tau(k)$ for every $k \in K$. From the fact that $N$ and $A_{\circ}$ are characteristic subgroups of $G$, one checks readily that $\beta \in A u t K$ and $\tau \in \operatorname{Hom}_{N}\left(K, A_{\circ}\right)$. Define $\delta: K \rightarrow A_{\circ}$ by $\delta(k)=\left(\tau \circ \beta^{-1}\right)\left(k^{-1}\right)$. It follows from $\beta(N)=N$ that $\delta \in \operatorname{Hom}_{N}\left(K, A_{\circ}\right)$; and hence, $\delta^{+} \in \operatorname{Aut}(G, K)$. Put $\epsilon=\delta^{+} \circ \alpha$. Since $\epsilon(k)=\beta(k) \tau(k) \delta(\beta(k))=\beta(k)$ for every $k \in K$, we have $\epsilon \in W(G, K)$. This proves $\operatorname{Aut}(G, K)=V(G, K) W(G, K)$. Moveover, suppose $\gamma \in \operatorname{Hom}_{N}\left(K, A_{\circ}\right)$. Then $\alpha^{-1} \circ \gamma \circ \beta \in \operatorname{Hom}_{N}\left(K, A_{\circ}\right)$. Because $A_{\circ}$ is contained in any maximal reductive complex Lie subgroup of $G$ and the difinition of $\gamma^{+}$is independent of any maximal reductive complex Lie subgroup of $G$, we have $\left(\alpha^{-1} \circ \gamma^{+} \circ \alpha\right)(k h)=\left(\alpha^{-1} \circ \gamma^{+}\right)(\beta(k) \tau(k) \alpha(h))=$ $\alpha^{-1}(\alpha(k h) \gamma(\beta(k)))=k h\left(\alpha^{-1} \circ \gamma \circ \beta\right)(k)$ for every $k \in K$ and $h \in H$; that is $\alpha^{-1} \circ$ $\gamma^{+} \circ \alpha=\left(\alpha^{-1} \circ \gamma \circ \beta\right)^{+}$. This proves that $V(G, K)$ is normal in $\operatorname{Aut}(G, K)$. Since $A_{\circ}$ is a characteristic subgroup of $G$, we see that $V(G, K)$ meets $W(G, K)$ trivially. This completes the proof.

Let $S=R(G, K)$ be the nuclear subalgebra of $R(G)$ that is associated with $K$ and $A_{S}$ the group of all proper automorphisms of $S$. Let $A_{S}=U \cdot M$, where $U$ is the unipotent radical of $A_{S}$ and $M$ is a maximal fully reducible subgroup of $A_{S}$. We shall identify $G$ as a closed complex analytic subgroup of $A_{S}$. Let $A u t A_{S}$ be the group of all complex analytic automorphisms of $A_{S}$. We are going to embed $\operatorname{Aut}(G, K)$ into Aut $A_{S}$. To do this, let $V_{1}\left(A_{S}\right)$ be the collection of all those $\alpha \in A u t A_{S}$ which leave $N \cdot M$ pointwise fixed and satisfy $\alpha(x) x^{-1} \in A_{\circ}$ for every $x \in U \cdot A_{\circ}$. It follows from Lemma 1.1 that $U \cdot A_{\circ}$ is the maximal nilpotent normal complex analytic subgroup of $A_{S}$. Thus, by [4, Theorem 1], $V_{1}\left(A_{S}\right)$ is a closed normal complex analytic subgroup of Aut $A_{S}$ and its canonical image in $G L\left(L\left(A_{S}\right)\right)$ is an algebraic subgroup of $G L\left(L\left(A_{S}\right)\right)$. 
Let $\operatorname{Hom}_{N}\left(U, A_{\circ}\right)$ be the collection of all complex analytic homomorphisms of $U$ into $A_{\circ}$ that are trivial on $N$. Let $\epsilon \in \operatorname{Hom}_{N}\left(U, A_{\circ}\right)$. Define $\epsilon^{*}: A_{S} \rightarrow A_{S}$ by $\epsilon^{*}(u m)=$ $u m \epsilon(u)$. Just like the case for $\operatorname{Hom}_{N}\left(K, A_{\circ}\right)$, one checks readily that $\epsilon^{*} \in A u t A_{S}$ and the definition of $\epsilon^{*}$ is independent of the choice of $M$. Let $V\left(A_{S}\right)$ be the collection of all $\epsilon^{*}$ as $\epsilon$ ranges over all elements of $\operatorname{Hom}_{N}\left(U, A_{\circ}\right)$. Since $U \cdot A$ is the maximal nilpotent normal subgroup of $A_{S}$ by Lemma 1.1, one concludes readily that $V\left(A_{S}\right)=V_{1}\left(A_{S}\right)$.

Lemma 2.2. $V\left(A_{S}\right)$ and $V(G, K)$ are isomorphic as complex analytic groups.

Proof. By [3, Theorem 3.1 and Theorem 4.1], there is a complex toroid $X$ such that $A_{S}=X \cdot G, M=X \cdot(M \cap G)$ is a direct product, $(X \cdot U) \cap G=K$ and $H=M \cap G$ is a maximal reductive complex Lie subgroup of $G$. (A complex Lie group is said to be a complex toroid if it is isomorphic with the complex analytic group $\left(\mathbf{C}^{*}\right)^{d}$ for some positive integer $d$.)

Let $\epsilon \in \operatorname{Hom}_{N}\left(U, A_{\circ}\right)$. If $k \in K$, we write $k=k_{U} k_{X}$ with $k_{U} \in U$ and $k_{X} \in X$, then $\epsilon^{*}(k)=k \epsilon\left(k_{U}\right) \in K A_{\circ}$. Together with the fact that $\epsilon^{*}$ is identity on $H$, we see that $\epsilon^{*} \mid G \in$ Aut $G$. Let $\gamma: K \rightarrow A_{\circ}$ be defined by $\gamma(k)=\epsilon\left(k_{U}\right)$. From the fact that $[X, U] \subset N$, one checks readily that $\gamma \in \operatorname{Hom}_{N}\left(K, A_{\circ}\right)$; and hence, $\gamma^{+} \in V(G, K)$. Since $\epsilon^{*}$ and $\gamma^{+}$coincide on $K$ and are both identity on $H$, we see that $\epsilon^{*} \mid G=\gamma^{+}$. It follows that the map $\psi: V\left(A_{S}\right) \rightarrow V(G, K)$ defined by $\psi\left(\epsilon^{*}\right)=\epsilon^{*} \mid G$, where $\epsilon \in \operatorname{Hom}_{N}\left(U, A_{\circ}\right)$, is a complex analytic homomorphism.

Suppose $\epsilon \in \operatorname{Hom}_{N}\left(U, A_{\circ}\right)$ such that $\epsilon^{*}$ is identity on $G$. Because $X \subset M, \epsilon^{*}$ is identity on $X$. Consequently, $\epsilon^{*}$ is identity on $A_{S}$. This shows that $\psi$ is injective. To see that $\psi$ is surjective, let $\gamma \in \operatorname{Hom}_{N}\left(K, A_{\circ}\right)$. Define $\epsilon_{1}: A_{S} \rightarrow A_{S}$ by $\epsilon_{1}(x k h)=x \gamma^{+}(k h)$ for $x \in X, k \in K$ and $h \in H$. From the facts that $X$ centralizes $H$ and $[M, K] \subset N$, one checks readily that $\epsilon_{1} \in$ Aut $A_{S}$. Since $\epsilon_{1}(U) \subset U A_{\circ}$ (by Lemma 1.1), by the definition of $\epsilon_{1}$, we see that there is an $\epsilon \in \operatorname{Hom}_{N}\left(U, A_{\circ}\right)$ such that $\epsilon_{1}(u)=u \epsilon(u)$ for all $u \in U$. Since $\epsilon_{1}$ and $\epsilon^{*}$ coincide on $U$ and both of them are identity on $M, \epsilon_{1}=\epsilon^{*}$ on $A_{S}$. Because $\gamma^{+}$is identity on $H$ and $\epsilon_{1}=\gamma^{+}$on $K$, we may conclude that $\psi\left(\epsilon^{*}\right)=\epsilon_{1} \mid G=\gamma^{+}$. This proves that $\psi$ is surjective and the proof of the lemma is complete.

Let $W\left(A_{S}\right)$ be the collection of all rational automorphisms of $A_{S}$ and $W\left(A_{S}, G, K\right)=$ $\left\{\alpha \in W\left(A_{S}\right): \alpha(G)=G, \alpha(K)=K\right\}$. Clearly, $W\left(A_{S}\right)$ and $W\left(A_{S}, G, K\right)$ are closed complex Lie subgroups of Aut $A_{S}$.

Lemma 2.3. $W(G, K)$ is isomorphic with $W\left(A_{S}, G, K\right)$ as complex Lie groups.

Proof. Let $\theta \in W(G, K)$. Clearly, $\theta$ induces an algebra automorphism ${ }^{t} \theta$ of $R(G)$ defined by ${ }^{t} \theta(f)=f \circ \theta$. Suppose that $B$ is a normal basic subalgebra of $R(G)$ such that $K$ is the kernel of the representation of $G$ by left translations on $B_{s}$. Since $\theta(K)=K$, one checks readily that ${ }^{t} \theta(B)$ is again a normal basic subalgebra of $R(G)$ that is associated with $K$. By [2, Theorem 4.1], ${ }^{t} \theta(B)=B \cdot x$ for some $x \in N$. Since ${ }^{t} \theta(S)$ is again a fully stable subalgebra of $R(G)$, it follows from the uniqueness of $S$ that ${ }^{t} \theta(S)=S$. Hence ${ }^{t} \theta$ induces an automorphism $\tilde{\theta}$ of the collection of all algebra homomorphisms of $S$ into C. In turn, $\tilde{\theta}$ induces an automorphism $\theta^{\#}$ of $A_{S}$ because $S$ is the algebra 
of polynomial functions on $A_{S}$. More precisely, if $\alpha \in A_{S}, f \in S$ and $x \in G$, then $\theta^{\#}(\alpha)(f)(x)=\alpha((f \cdot x) \circ \theta)(e)$, where $e$ is the identity element of $G$. Clearly, $\theta^{\#}$ leaves $G$ and $K$ invariant. Together with the result ${ }^{t} \theta(S)=S$, we see that $\theta^{\#} \in$ $W\left(A_{S}, G, K\right)$. It is then straightforward to check that the map $\theta \mapsto \theta^{\#}$ is a complex analytic homomorphism of $W(G, K)$ into $W\left(A_{S}, G, K\right)$. Since $S$ separates points of $G$, one sees readily that the map $\theta \mapsto \theta^{\#}$ is injective. Let $\phi \in W\left(A_{S}, G, K\right)$. Clearly, $\phi \mid G \in W(G, K)$. Then it is straightforward to check that $(\phi \mid G)^{\#}=\phi$ on $G$. Since $(\phi \mid G)^{\#}$ and $\phi$ are rational automorphisms on $A_{S}$ and $G$ is algebraically dense in $A_{S}$, we may conclude that $(\phi \mid G)^{\#}=\phi$ on $A_{S}$. This proves that the map $\theta \mapsto \theta^{\#}$ is surjective and the proof of the lemma is complete.

Theorem 2.4. Let $G$ be a faithfully representable complex analytic group, $K$ a nucleus of $G, S=R(G, K)$ and $A_{S}$ the group of all proper automorphisms of $S$. Then Aut $(G, K)_{\circ}$ can be identified as an algebraic subgroup of $\left(\text { Aut } A_{S}\right)_{\circ}$.

Proof. By [4, Theorem 1 and Theorem 3], Aut $A_{S}$ is a semidirect product $V\left(A_{S}\right)$. $W\left(A_{S}\right), V\left(A_{S}\right)$ is a connected algebraic group and (Aut $\left.A_{S}\right)_{\circ}=V\left(A_{S}\right) \cdot W\left(A_{S}\right)_{\circ}$ is an algebraic group. Clearly, $E=\left\{\alpha \in W\left(A_{S}\right)_{\circ}: \alpha(G)=G\right.$ and $\left.\alpha(K)=K\right\}$ is an algebraic subgroup of $W\left(A_{S}\right)$ 。 and $E_{\circ}=W\left(A_{S}, G, K\right)_{\circ}$; and hence, $W\left(A_{S}, G, K\right)$ 。 is an algebraic group. Together with Theorem 2.1, Lemma 2.2 and Lemma 2.3, we may conclude that $\operatorname{Aut}(G, K) \circ=V(G, K) \cdot W(G, K)$ 。 is isomorphic with the algebraic subgroup $V\left(A_{S}\right) \cdot W\left(A_{S}, G, K\right)$ 。 $W\left(A_{S}\right)_{\circ}$ as complex analytic groups. This completes the proof.

Let $J$ be the maximal nilpotent normal subgroup of the radical $R$ of $G$. By Theorem 1.2 , there is a nucleus $K$ of $G$ that is contained in $R$ such that $J=(J \cap K) \cdot(J \cap A)$ and $\theta(K) \subseteq K A_{\circ}$ for all $\theta \in A n t G$. Together with Theorem 2.4, we have our main result.

Theorem 2.5. Let $G$ be a faithfully representable complex analytic group. Then $(\text { Aut } G)_{\circ}$ can be identified as an algebraic subgroup of $\left(\text { Aut } A_{S}\right)_{\circ}$, where $K$ is a nucleus of $G$ such that $\theta(K) \subseteq K A_{\circ}$ for all $\theta \in A n t G$, and $S$ is the nuclear subalgebra of $R(G)$ that is associated with $K$.

As a corollary, we have the following.

Corollary 2.6. Let $G$ be a faithfully representable complex analytic group, $x \in G$ and Aut $_{\circ}(G,\{x\})=\left\{\alpha \in(\text { Aut } G)_{\circ}: \alpha(x)=x\right\}$. Then Aut $_{\circ}(G,\{x\})$ can be identified as an algebraic subgroup of $\left(\text { Aut } A_{S}\right)_{\circ}$, where $K$ is a nucleus of $G$ such that $\theta(K) \subseteq K A_{\circ}$ for all $\theta \in A n t G$, and $S$ is the nuclear subalgebra of $R(G)$ that is associated with $K$.

Proof. Fix a maximal reductive complex Lie subgroup $H$ of $G$ and write $x=k h$ with $k \in K$ and $h \in H$. Let $\alpha \in A u t_{\circ}(G,\{x\})$. By Theorem 2.1 and Theorem 2.4, $\alpha=\gamma \alpha^{+} \circ \beta_{\alpha}$ with $\gamma_{\alpha} \in \operatorname{Hom}_{N}\left(K, A_{\circ}\right)$ and $\beta_{\alpha} \in W(G, K)_{\circ}$. Since $\alpha(x)=x$, we have $k h=\gamma \alpha^{+}\left(\beta_{\alpha}(k h)\right)=\beta_{\alpha}(k h) \gamma_{\alpha}\left(\beta_{\alpha}(k)\right)$, which implies $\beta_{\alpha}(x) x^{-1}=\gamma_{\alpha}\left(\beta_{\alpha}\left(k^{-1}\right)\right)=$ $\beta_{\alpha}(k) \gamma_{\alpha}^{+}\left(\beta_{\alpha}(k)^{-1}\right)$. This proves that $\operatorname{Aut}_{\circ}(G,\{x\})$ consists of all those elements $\alpha \in$ (Aut $G)$ 。 for which $\beta_{\alpha}(x) x^{-1}=\beta_{\alpha}(k) \gamma_{\alpha}^{+}\left(\beta_{\alpha}(k)^{-1}\right)$. By Theorem 2.5, (AutG)。can be 
identified as an algebraic subgroup of $\left(\operatorname{Aut} A_{S}\right)_{\circ}$. It follows that $\operatorname{Aut}_{\circ}(G,\{x\})$ can be identified as an algebraic subgroup of $\left(\text { Aut } A_{S}\right)_{\circ}$. This completes the proof.

\section{References}

[1] P. B. Chen and T. S. Wu, Hopf algebras, Lie algebras, and analytic groups, J. Algebra 181(1996), 1-15.

[2] G. Hochschild and G. D. Mostow, On the algebra of representative functions of an analytic group, Amer. J. Math. 83(1961), 111-136.

[3] G. Hochschild and G. D. Mostow, On the algebra of representative functions of an analytic group II, Amer. J. Math. 86(1964), 869-887.

[4] G. Hochschild and G. D. Mostow, Analytic and rational automorphisms of complex algebraic groups, J. Algebra 25(1973), 146-151.

Department of Mathematics and Computer Science, John Carroll University, University Heights, OH 44118.

Department of Mathematics and Statistics, Case Western Reserve University, Cleveland, OH 44106 . 